\title{
Comparación de etapas de degradación vegetacional con manejo pecuario utilizando valores bioindicadores de Ellenberg en la Patagonia Chilena
}

\author{
Comparison of vegetation stages degradation with livestock management using Ellenberg's \\ bioindicators in Chilean Patagonia
}

\author{
Cristina San Martín a*, Víctor Sandoval b, Miguel Álvarez ${ }^{\text {c}}$, Osvaldo Vidal d, Yéssica Pérez a José L Solís a \\ *Autor de correspondencia: ${ }^{a}$ Universidad Austral de Chile, Instituto de Ciencias Ambientales y Evolutivas, \\ casilla 567, Valdivia, Chile, teléfono 56-63-2221511, csanmart@uach.cl

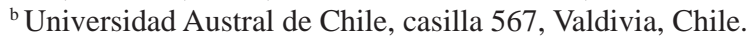 \\ c Reinische Friedrich-Wilhelms-Universität, INRES-Vegetationsökologie, Bonn, Alemania. \\ ${ }^{d}$ Universidad de Magallanes, Instituto de la Patagonia, Avenida Bulnes 01855, Punta Arenas, Chile.
}

\begin{abstract}
SUMMARY
Different stages of anthropogenic degradation series of one primary shrub were compared in Aisén, Chile. It consisted of a prolonged livestock pasture, with plant ecological indicators recognized and valued by Ellenberg for the factors of light, temperature and reaction, nitrogen and soil moisture. The primary ñirre thicket was considered the beginning of the series. We worked with partial vegetation tables for each state of degradation. Indicator plants as well as nature and utility assessed by multivariate statistical methods for classification and ordination are identified in these tables. Comparison among communities was carried out considering the presence and abundance of selected indicator plants. Results indicate that light and temperature values are concentrated in some parts of the scale from 1 to 9 steps, proposed by Ellenberg; while the others, more dispersed, provide a better differentiation of degradation states. It was possible to characterize the biotopes of all stages of degradation as: sunny, presenting mild temperatures with occasional frost and acid soils, deficient in nutrients and with intermediate moisture. The greatest differentiation was obtained with the latter soil variables that coincided with the position of each of them in the degradation series. This was confirmed by soil tests.
\end{abstract}

Key words: indicators plants, degradation stages, pasture, Aisén, Chile.

\section{RESUMEN}

En Aisén, Chile, se compararon distintos estadios de degradación antropogénica de la vegetación arbustiva original en una serie de distintos manejos de pastoreo, utilizando los indicadores ecológicos vegetales de Ellenberg para los factores de luz, temperatura del aire y de reacción, nitrógeno y humedad del suelo. El matorral de ñirre primario se consideró como inicio de la serie. Se trabajó con tablas de vegetación separadas para cada estado de degradación. En estas tablas se identificaron especies indicadoras, cuya naturaleza y utilidad fue corroborada mediante métodos estadísticos multivariados de clasificación y de ordenación. La comparación entre las comunidades se realizó considerando la presencia y la abundancia de las plantas indicadoras presentes en ellas. Los resultados indican que los valores de luz y temperatura se concentran en algunos tramos de la escala propuesta por Ellenberg, mientras que los restantes, más dispersos, entregan una mejor diferenciación de los estados de degradación. Se pudo caracterizar los biotopos de todos los estados de degradación como de sol, de temperaturas templadas con heladas ocasionales y de suelos ácidos, deficientes en nutrientes y con humedad intermedia. La mayor diferenciación se obtuvo en estas últimas variables edáficas que entregaron una ordenación de las comunidades coincidente con la posición de cada una de ellas en la serie de degradación descrita con anterioridad, esto fue confirmado con los análisis de suelo.

Palabras clave: plantas indicadoras, series de degradación, pastoreo, Aisén, Chile.

\section{INTRODUCCIÓN}

El paisaje del centro-sur de Chile está formado por comunidades vegetales primarias, secundarias y terciarias. Las primeras son originales del lugar y forman la vegetación "potencial", las segundas son comunidades de remplazo de la vegetación primaria, forzadas por el hombre para producir alimentos, las terciarias invaden estas última cuando son degradas por el mal manejo, (Ramírez et al.
1992). Rodales de todas estas comunidades conviven actualmente en el paisaje chileno, siendo difícil identificar la posición que ocupan en las series de degradación. Esta dinámica de vegetación es importante porque refleja la huella de las actividades productivas, permitiendo la reconstrucción teórica del proceso de degradación y la restauración práctica de las condiciones originales. Ramírez et al. (2012) estudiaron una sucesión secundaria en la región de Aisén (Chile), que se inicia en un matorral primario de 
ñirre (Nothofagus antarctica) ${ }^{1}$, continúa con un silvopastoreo bajo el mismo matorral, intervención que culmina en una pradera fresca de trébol-ballica (Trifolium repensLolium perenne), pero luego en esta, por sobrepastoreo, se forma una comunidad pratense degradada de cadilloaira (Acaena integerrima-Aira caryophyllea), que finaliza transformándose en una estepa terciaria de cadillo (Acaena integerrima), improductiva para el hombre. Además existen un silvopastoreo con chacay (Discaria chacaye) y una pradera de chépica-cadillo (Agrostis capillaris-Acaena ovalifolia) desviadas de la serie principal.

Las malezas herbáceas que acompañan al hombre en el proceso de degradación del paisaje han convivido con cultivos y formaciones pratenses desde el inicio de la agricultura y la ganadería, adaptándose a los continuos cambios antropogénicos del hábitat. Por lo anterior, tienen una gran aplicación como indicadoras de esos cambios.

Para cuantificar la evaluación de biotopos agropecuarios se usan los "valores indicadores ecológicos" propuestos por Ellenberg et al. (1992), con una escala ordinal de nueve tramos para los factores de luz y temperatura (factores microclimáticos) y reacción (pH), nitrógeno y humedad del suelo (factores edáficos), en la cual, el valor 1 indica escasa afinidad con el factor y el 9 una alta afinidad y dependencia de él, considerando el 5 como intermedio. La indiferencia a los factores se indica con 0 . Prácticamente toda la flora de malezas chilenas bioindicadoras se encuentra en catálogos europeos y un resumen de ella fue recopilado por Ramírez et al. (1991). Ellas facilitan la comparación entre manejos sin necesidad de realizar costosas y largas mediciones microclimáticas y análisis de suelos, ya que corresponden en forma aproximada a los requerimientos de las especies utilizadas como indicadoras. Los valores indicadores son ordinales y carecen de unidad de medida.

El objetivo del presente estudio es comparar las comunidades vegetales en la serie de degradación comentada, utilizando los bioindicadores de Ellenberg. La hipótesis de trabajo supone que con ayuda de estos bioindicadores vegetales alóctonos es posible caracterizar los hábitats que ofrece cada una de las comunidades de la serie de degradación descrita por Ramírez et al. (2012), no siendo necesario agregar especies indicadoras nativas, ya que ellas caracterizan mejor la vegetación primaria original, antes que los distintos manejos antrópicos, en los que van desapareciendo.

\section{MÉTODOS}

La base del presente estudio fue la tabla final preparada por Ramírez et al. (2012) ordenada por un valor de importancia calculado utilizando el método de Wikum y Schanholtzer (1978). En dicha tabla formada por 77 especies vegetales y 50 censos de vegetación, se seleccionaron especies indicadoras según la literatura (Ellenberg et al. 1992, Ramírez et al. 1991), a las cuales se determinó el origen

Autores botánicos de las especies en el cuadro 1. (autóctono o alóctono) (Zuloaga et al. 2008) ponderándolas tanto por el número de especies como por la cobertura (cuadro 1). En los resultados las especies serán designadas con sus nombres científicos y las comunidades con aquellos vernáculos.

La misma tabla ordenada fue sometida a clasificación mediante análisis de conglomerados y a ordenación por análisis de componentes principales. En el primer caso fue aplicado el algoritmo de la media UPGMA (método de agrupamiento no ponderado utilizando medias aritméticas) considerando la distancia euclidiana y datos no transformados. En el análisis de componentes principales se utilizaron datos de frecuencia transformados para homogenizar la varianza. Ambos métodos buscaban encontrar la agrupación y posición de las especies indicadoras seleccionadas en relación con el resto no indicador (Leyer y Wesche 2007).

La tabla ordenada fue desagregada en sus comunidades, para formar tablas parciales de cada una (cuadro 2). En ellas se colocaron sólo las especies indicadoras de cada manejo (comunidad) con sus correspondientes valores para los factores de luz, temperatura, reacción, nitrógeno y humedad (Álvarez et al. 2013). Con estos valores se calcularon en cada comunidad promedios para cada factor, dividiendo la suma de ellos por el número de especies indicadoras, el resultado fue tratado como valor indicador promedio por presencia de especies. Para obtener estos promedios ponderando la abundancia de los individuos, se multiplicó el valor indicador por la cobertura de cada especie y luego la suma se dividió por la cobertura total de las especies de la comunidad. Mayores detalles de esta metodología se encuentran en Ramírez et al. (1992) y Álvarez et al. (2013).

Para comparar los resultados de los factores referidos a las características edáficas se tomó una muestra superficial (hasta $10 \mathrm{~cm}$ ) de suelo en sectores homogéneos y representativos de cada comunidad las que fueron procesadas en el Instituto de Ingeniería Agraria y Suelos de la Facultad de Ciencias Agrarias de las Universidad Austral de Chile utilizando metodologías estandarizadas para determinar $\mathrm{pH}$ (reacción del suelo), materia orgánica, nitrógeno mineral, fósforo Olsen y calcio intercambiable.

\section{RESULTADOS}

De las 77 especies vegetales presentes en la tabla de vegetación formada por los manejos estudiados, 33 (42,9 \%) han sido reconocidas como indicadores de condiciones del hábitat (cuadro 1). En dicha tabla 377 casillas son ocupadas por las especies indicadoras (68,8 \%), mientras que las no evaluadas aún en su carácter de indicador comprendieron el $31,2 \%$ restante. Al considerar la abundancia de los individuos, el peso de las especies indicadoras sube a 70,4 \% y él de las no indicadoras descienden a 29,6\%. De las 33 especies reconocidas como indicadoras, la mayoría son malezas alóctonas (81,8 \%), las restantes (18,2 \%) son autóctonas. 
Cuadro 1. Especies de la tabla inicial ordenadas por el valor de importancia de mayor a menor. Las especies señaladas con el signo * son las utilizadas como indicadoras.

Species of the initial table sorted by importance value, from the highest to the lowest. Species indicated by * are used as indicators.

\begin{tabular}{|c|c|c|c|}
\hline Especies & $\begin{array}{c}\text { Valor de } \\
\text { importancia }\end{array}$ & $\begin{array}{l}\text { Chilliotrichum rosmarinifolium Less. } \\
\text { Musgos }\end{array}$ & $\begin{array}{l}0,60 \\
0,60\end{array}$ \\
\hline *Taraxacum officinale Webb. & 25,61 & Ribes trilobum Meyen & 0,58 \\
\hline *Agrostis capillaris L. & 18,21 & Deschampsia flexuosa (L.) Trin. & 0,48 \\
\hline Acaena integerrima Gill. ex Hook et Arn. & 16,98 & Trisetum spicatum (L.) K.Richt. & 0,48 \\
\hline *Holcus lanatus L. & 13,45 & Maytenus disticha (Hook.f.) Urb. & 0,44 \\
\hline *Achillea millefolium L. & 11,45 & Escallonia virgata (Ruiz et Pav.) Pers. & 0,40 \\
\hline *Trifolium repens L. & 10,15 & Gavilea lutea (Pers.) M.N.Correa & 0,40 \\
\hline Nothofagus antarctica (G.Forst.) Oerst. & 10,02 & Gaultheria mucronata (L.f) Hook. et Arn. & 0,40 \\
\hline *Aira praecox L. & 9,64 & Geranium sessiliflorum Cav. & 0,40 \\
\hline *Poa pratensis L. & 7,71 & Lathyrus magellanicus Lam. & 0,40 \\
\hline *Rumex acetosella L. & 5,85 & Misodendron punctulatum Banks. ex DC. & 0,40 \\
\hline *Cerastium arvense L. & 4,84 & Phacelia secunda J.F.Gmel. & 0,40 \\
\hline Discaria chacaye (G. Don)Tortosa & 4,13 & *Plantago major L. & 0,40 \\
\hline Anemone multifida Poir. & 3,87 & *Rumex conglomeratus Murr. & 0,40 \\
\hline *Hypochaeris radicata $\mathrm{L}$. & 3,34 & Viola maculata Cav. & 0,40 \\
\hline Berberis microphylla G. Forst. & 3,32 & *Anagallis alternifolia Cav. & 0,37 \\
\hline *Fragaria chiloensis (L.) Duch. & 3,30 & Phleum alpinum L. & 0,37 \\
\hline *Lolium perenne L. & 3,27 & Empetrum rubrum Vahl. Ex Willd. & 0,28 \\
\hline *Carex fuscula D’Urv. & 2,58 & Azorella trifurcata (Gaertn.) Pers. & 0,20 \\
\hline Acaena pinnatifida Ruiz et Pav. & 2,42 & Baccharis magellanica (Lam.) Pers. & 0,20 \\
\hline *Crepis capillaris (L.) Wallr. & 2,38 & *Bromus catharticus Vahl. & 0,20 \\
\hline *Plantago lanceolata L. & 2,29 & *Cerastium glomeratum Thuill. & 0,20 \\
\hline Blechnum penna-marina (Poir.) Kuhn. & 2,24 & Chlorea magellanica Hook.f. & 0,20 \\
\hline *Aira caryophyllea L. & 2,22 & Chusquea culeou E. Desv. & 0,20 \\
\hline *Centaurium littorale (Turner) Gilmour. & 1,81 & *Carduus nutans L. & 0,20 \\
\hline *Vulpia bromoides (L.) Gray & 1,81 & Collomia biflora (Ruiz et Pav.) Brand. & 0,20 \\
\hline Prunella vulgaris L. & 1,61 & Colobanthus quitensis (Kunth) Bartl. & 0,20 \\
\hline *Acaena ovalifolia Ruiz et Pav. & 1,57 & Euphrasia antarctica Benth. & 0,20 \\
\hline Geranium berteroanum Colla & 1,41 & *Galium aparine L. & 0,20 \\
\hline *Rosa rubiginosa L. & 1,41 & Hieracium chilense Less. & 0,20 \\
\hline *Cirsium vulgare (Savi) Ten. & 1,36 & Juncus microcephalus Kunth. & 0,20 \\
\hline *Trifolium pratense L. & 1,33 & Microsteris gracilis (Hook.) Greene & 0,20 \\
\hline Osmohriza berteroi DC. & 1,01 & Misodendron linearifolium DC. & 0,20 \\
\hline *Dactylis glomerata L. & 0,98 & *Myosotis stricta Link. ex Roem. et & \\
\hline Geum magellanicum Pers. & 0,81 & Schult. & 0,20 \\
\hline Quinchamalium chilense Molina & 0,81 & *Ranunculus repens L. & 0,20 \\
\hline Myoschilos oblonga Ruiz et Pav. & 0,68 & Ribes cucullatum Hook et Arn. & 0,20 \\
\hline Mutisia spinosa Ruiz et Pav. & 0,64 & Rubus geoides Sm. & 0,20 \\
\hline Acaena magellanica (Lam.) Vahl. & 0,60 & *Vicia nigricans Hook. et Arn. & 0,20 \\
\hline Chilliotrichum diffusum (G. Forst.) Kunze & 0,60 & (Total especies 78) / Total: & 200 \\
\hline
\end{tabular}


Cuadro 2. Comunidades y el correspondiente nombre científico de la asociación considerada en la serie de degradación con la abreviatura utilizada en las figuras 6,7 y cuadro 8. Las 5 comunidades superiores forman la serie de degradación que aumenta hacia abajo y las 2 de abajo, son comunidades que se desvían de ella.

Communities and the scientific name for the association of degradation series with the abbreviation used in figures 6,7 and table 8 . The upper 5 communities form the degradation series which increase downwards and the last 2 are communities that deviate from it.

\begin{tabular}{lcl}
\hline Comunidad & Abreviatura & Asociación \\
\hline Matorral de ñirre & MÑ & Anemono-Nothofagetum antarcticae \\
Silvopastoreo con ñirre & SÑ & Estado de degradación \\
Pradera de trébol-ballica & PT-B & Taraxaco-Trifolietum repentis \\
Pradera de cadillo-aira & PC-A & Airo-Acaenetum integerrimae \\
Estepa de cadillo & EC & Acaenetum ingerrimae \\
Silvopastoreo con chacay & SC & Estado de degradación \\
Pradera de chépica-cadillo & PC-C & Acaeno-Agrostietum capillariae \\
\hline
\end{tabular}

Comparación multivariada de especies y comunidades. El dendrograma de afinidad entre las especies indica que la mayoría nativa considerada no indicadora, presentan alta similitud entre ellas, ubicándose en la parte central (figura 1). Por el contrario, las especies indicadoras tienden a ubicarse en los extremos del gráfico demostrando poca afinidad con las otras y requerimientos propios lo que caracteriza a las especies diferenciales de grupos de vegetación e indicadoras de condiciones del hábitat. Junto a ellas y en el extremo inferior del dendrograma aparecen algunos arbustos nativos (Berberis microphylla, Discaria chacaye y Nothofagus antarctica) y un subarbusto (Acaena integerri$\mathrm{ma}$ ) especie que coloniza suelos desnudos y degradados. La posición de estas últimas especies señala que también podrían considerarse como indicadoras.

Lo anterior coincide con la posición que toman estas especies en el plano formado por los dos primeros componentes principales que reúnen el 41,6 \% de la variación (figura 2). La gran mayoría de las especies se concentra en el centro del gráfico y sólo las plantas alóctonas indicadoras de mayor importancia, se segregan ocupando situaciones más extremas. Muy distanciadas entre sí en los extremos del segundo eje vertical, aparecen Acaena integerrima y Agrostis capillaris, la primera coloniza biotopos altamente degradados y erosionados, la segunda ocupa hábitats pratenses abandonados con cubierta vegetal continua. Por el primer componente horizontal se separan, aunque por una menor distancia, Cerastium arvense y Rumex acetosella de Taraxacum officinale. Los primeros aparecen en ambientes más luminosos y secos, y el último en condiciones de mayor sombra y humedad, soportando pisoteo y pastoreo.

Especies indicadoras. Las comunidades con mayor cantidad de indicadores corresponden al silvopastoreo con chacay, pradera de trébol-ballica y pradera de chépica-cadillo, facilitando posiblemente una mayor diferenciación de sus biótopos (cuadro 3). Con menor número de especies indicadoras figuran el silvopastoreo con ñirre y la pradera de cadillo-aira.
Posiciones intermedias ocupan el matorral de ñirre y la estepa de cadillo. La mayor cantidad de indicadoras podría estar señalando una mayor alteración de los biotopos, ya que la mayoría de ellas corresponde a malezas introducidas, además, se constató una débil correlación positiva entre el número total de especies en la comunidad y el número de indicadoras.

El número de especies indicadoras (cuadro 4) corresponde al $43 \%$ del total de especies presentes en la tabla ordenada. De este total, 14 (42 \%) son indiferentes a más de un factor, nueve especies son indiferentes a un solo factor, 2 a 2 y 3 a 3 factores (indicadas con 0 en el cuadro 4).

El número de especies no indicadoras e indiferentes a uno o más factores en cada comunidad presentó una estrecha afinidad con la cantidad total de indicadoras presentes en cada una de ellas (figura 3). Lo anterior señala que a mayor cantidad de especies indicadoras aumenta la posibilidad de encontrar especies indiferentes a algún factor.

La mayoría de las especies se dispersa en toda la escala de cada factor, sin embargo, la luz y la temperatura se concentran en un valor determinado, en el primer caso en valores altos y en el segundo, en valores intermedios (figura 4). Los altos valores de luz, coinciden en el hecho de que las malezas indicadoras son hierbas poco tolerantes que necesitan mucha luz para su desarrollo, la concentración intermedia de las especies por temperatura está de acuerdo con el clima templado frío de la región trabajada. En todo caso estos dos factores de tipo microclimático, no deberían provocar mayor diferenciación entre las comunidades estudiadas, ya que todas ellas se ubican en un mismo macroclima y en lugares próximos entre sí. Los otros factores presentan una mayor dispersión y no muestran barras altas que destaquen, esto indica que la mayor diferenciación de los biotopos se produce por el efecto de ellos, que corresponden a variables edáficas, frecuentemente alteradas por los diferentes manejos del suelo.

Valores indicadores promedios por presencia. Al considerar solo el número de especies indicadoras en cada comu- 


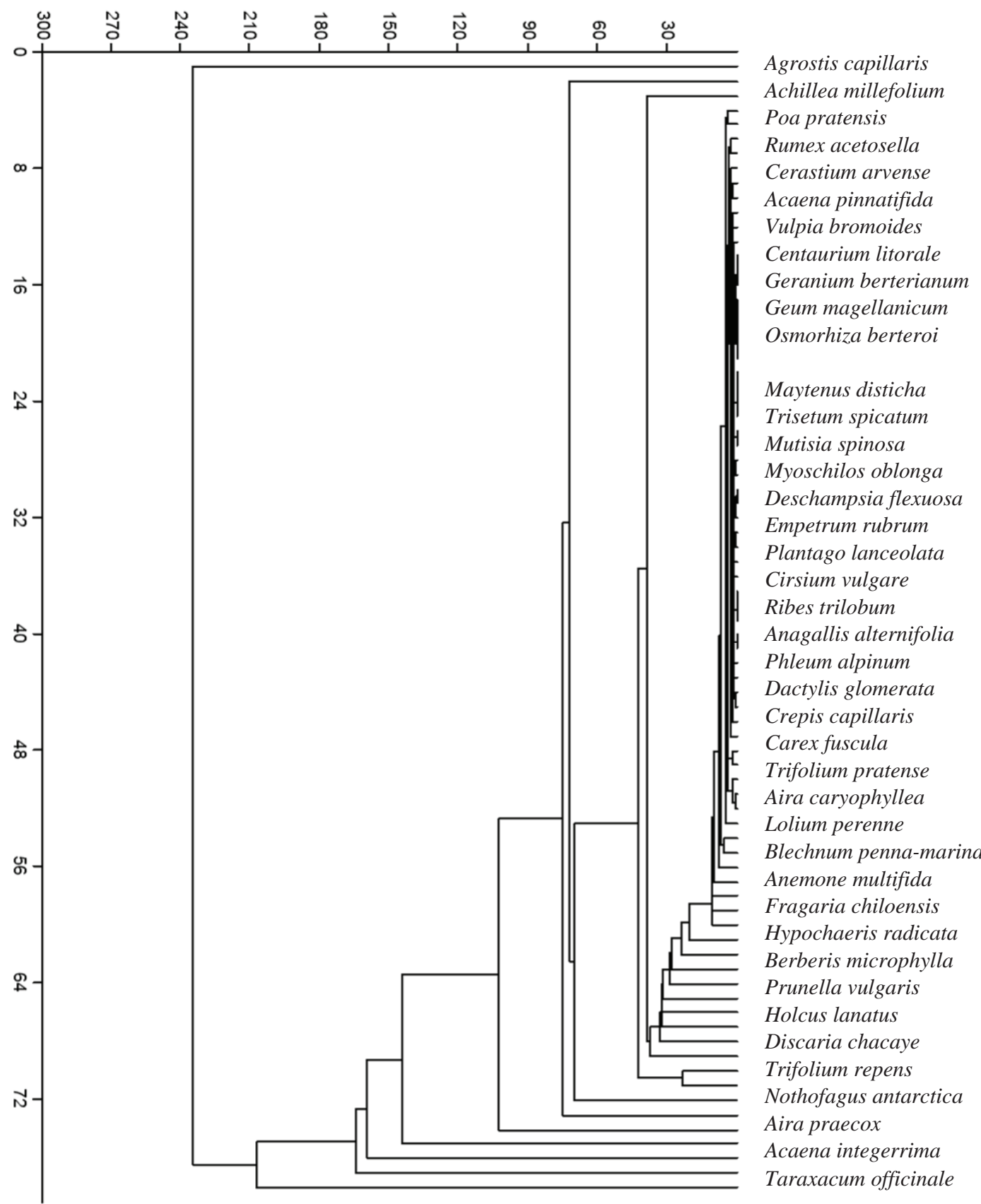

Figura 1. Análisis de conglomerado UPGMA que muestra la agrupación de las especies de la tabla inicial utilizando la distancia Euclidiana sobre los datos sin transformar. Las especies indicadoras de mayor peso aparecen en los extremos del diagrama.

UPGMA cluster analysis showing clustering of the species of the initial table using the Euclidean distance on raw data. Indicator species are located at the ends of the diagram.

nidad la luz alcanza el valor promedio más alto $(7,82)$, que indica plantas con una afinidad muy cercana a especies que crecen a plena luz (cuadro 5), como efectivamente son las malezas introducidas utilizadas como indicadores biológicos. En este caso la variación fue mínima (3,4 \%) ya que el valor máximo alcanzó a 8,17 en la estepa de cadillo y el mínimo de 7,40 en el silvopastoreo con ñirre. Para el factor temperatura el promedio fue de 5,52 propio de climas moderados de tierras bajas, pero que pueden soportar heladas.
En este caso la variación alcanzó a un 10,08 \% con un valor máximo de 6,63 en la pradera de trébol-ballica y un mínimo de 4,75 en el silvopastoreo con ñirre que vendría a ser el biotopo más frío y sombrío.

El promedio indicador para la reacción $(\mathrm{pH})$ del suelo resultó de 4,57 lo que corresponde a suelos poco ácidos, el mayor valor se encontró en el silvopastoreo con chacay y los menores en el silvopastoreo con ñirre y en la pradera de aira-cadillo, siendo la variación de 12,15 \%, la más alta 


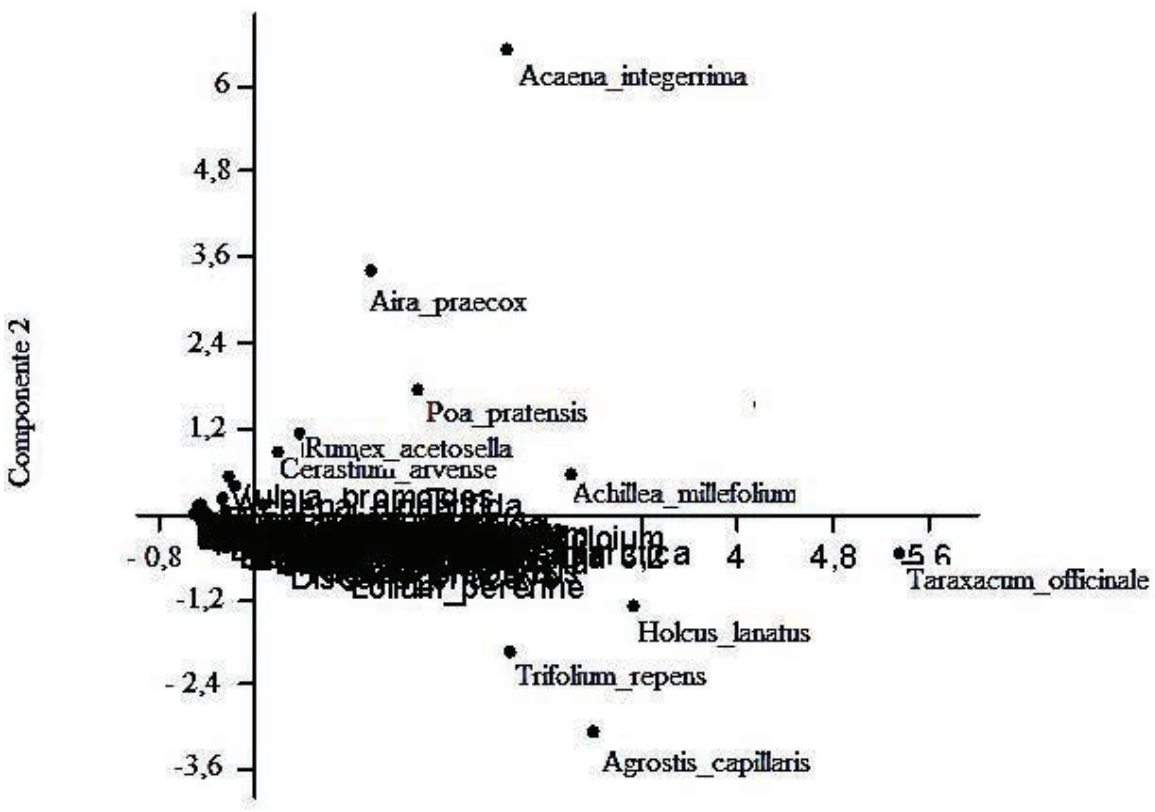

Componente 1

Figura 2. Distribución de las especies en el plano formado por los dos primeros componentes principales, que explican el 41,6 \% de la varianza. Las especies indicadoras se segregan hacia los bordes del plano, las no indicadoras se agrupan en el centro de la figura. Species distribution in the plane formed by the first two principal components, which account for $41.6 \%$ of the variance. Indicator species are segregated to the edges of the plane; non-indicators are grouped in the center of the figure.

Cuadro 3. Total de especies indicadoras e indiferentes a algún factor por comunidad, el porcentaje entre paréntesis se refiere a la columna inmediatamente anterior en cada caso.

Total of indicator and indifferent species to any factor by community; the percentage in parentheses refers to the preceding column in each case.

\begin{tabular}{lccc}
\hline Comunidades / Especies: & Total especies & Indicadoras & Indiferentes \\
\hline Matorral de ñirre & 43 & $12(27,90)$ & $6(50,00)$ \\
Silvopastoreo con ñirre & 11 & $5(45,45)$ & $4(80,00)$ \\
Silvopastoreo con chacay & 24 & $15(62,50)$ & $7(46,67)$ \\
Pradera de trébol-ballica & 29 & $19(65,52)$ & $8(42,11)$ \\
Pradera de chépica-cadillo & 36 & $23(63,89)$ & $9(30,13)$ \\
Pradera de cadillo-aira & 13 & $8(61,54)$ & $4(50,00)$ \\
Estepa de cadillo & 19 & $12(63,16)$ & $6(50,00)$ \\
\hline
\end{tabular}

medida al considerar sólo la presencia de indicadoras, lo que indica alteración del contenido en nutrientes del suelo. El valor promedio para el factor nitrógeno alcanzó a 4,77 cercano a los niveles considerados intermedios, en este caso la variación fue un poco menor que la reacción del suelo llegando a 8,32 \% con un máximo de 5,20 en el silvopastoreo con ñirre y un mínimo de 4,25 en la pradera de cadillo-aira. Por último, el promedio del factor indicador humedad alcanzó a 4,68, que corresponde a condiciones de suelos frescos con niveles de humedad intermedia, el valor más alto $(5,25)$ se presentó en el silvopastoreo con ñirre y el menor $(4,20)$ en la estepa de cadillo, un biotopo con condiciones esteparias xéricas y poca materia orgánica en el suelo, con una variación de 7,86 \%.

Valores indicadores promedios por cobertura. Al ponderar la abundancia (cobertura) de los individuos de las especies indicadoras se observaron pocos cambios. Los valores promedio para los indicadores de luz y de nitrógeno subieron en un 0,09 para el primero y un 0,15 el segundo, confirmando en el primer caso el carácter luminoso de los hábitats y en el segundo, la presencia de niveles interme- 
Cuadro 4. Valores indicadores para los factores luz (L), temperatura (T), reacción (R), nitrógeno (N) y humedad (H) de cada especie indicadora según Ramírez et al. (1991). La indiferencia se representa con un cero (0).

Indicator values for the factors light $(\mathrm{L})$, temperature $(\mathrm{T})$, reaction $(\mathrm{R})$, nitrogen $(\mathrm{N})$ and humidity $(\mathrm{H})$ of each indicator species after Ramírez et al. (1991). Indifference is represented by a zero (0).

\begin{tabular}{llllll}
\hline \multirow{2}{*}{\multicolumn{1}{c}{ Especies }} & \multicolumn{5}{c}{ Valores indicadores de Ellenberg } \\
\cline { 2 - 6 } & $\mathrm{L}$ & $\mathrm{T}$ & $\mathrm{R}$ & $\mathrm{N}$ & $\mathrm{H}$ \\
\hline Acaena ovalifolia & 6 & 4 & 2 & 3 & 5 \\
Achillea millefolium & 8 & 4 & 0 & 5 & 4 \\
Agrostis capillaris & 7 & 5 & 3 & 4 & 0 \\
Aira caryophyllea & 9 & 8 & 4 & 4 & 3 \\
Aira praecox & 9 & 6 & 2 & 1 & 2 \\
Anagallis alternifolia & 7 & 7 & 8 & 7 & 9 \\
Bromus catharticus & 9 & 5 & 6 & 5 & 0 \\
Carduus nutans & 8 & 6 & 8 & 6 & 4 \\
Carex fuscula & 7 & 5 & 4 & 5 & 8 \\
Centaurium littorale & 9 & 7 & 6 & 5 & 6 \\
Cerastium arvense & 9 & 0 & 6 & 4 & 5 \\
Cerastium glomeratum & 7 & 5 & 5 & 5 & 5 \\
Cirsium vulgare & 8 & 6 & 7 & 8 & 4 \\
Crepis capillaris & 7 & 6 & 7 & 4 & 4 \\
Dactylis glomerata & 7 & 5 & 7 & 7 & 5 \\
\hline
\end{tabular}

\begin{tabular}{llllll}
\hline Fragaria chiloensis & 8 & 6 & 6 & 3 & 4 \\
Galium aparine & 7 & 6 & 6 & 8 & 0 \\
Holcus lanatus & 7 & 5 & 0 & 4 & 6 \\
Hypochaeris radicata & 8 & 5 & 2 & 4 & 5 \\
Lolium perenne & 8 & 5 & 0 & 6 & 5 \\
Myosotis stricta & 8 & 6 & 6 & 2 & 3 \\
Plantago lanceolata & 7 & 5 & 0 & 6 & 0 \\
Plantago major & 9 & 0 & 0 & 0 & 4 \\
Poa pratensis & 7 & 5 & 7 & 7 & 5 \\
Ranunculus repens & 7 & 0 & 0 & 0 & 7 \\
Rosa rubiginosa & 9 & 6 & 5 & 5 & 3 \\
Rumex acetosella & 8 & 5 & 1 & 2 & 5 \\
Rumex conglomeratus & 8 & 5 & 5 & 8 & 7 \\
Taraxacum officinale & 8 & 0 & 0 & 7 & 5 \\
Trifolium pratense & 8 & 5 & 7 & 7 & 0 \\
Trifolium repens & 8 & 5 & 6 & 6 & 0 \\
Vicia nigricans & 5 & 6 & 0 & 0 & 0 \\
Vulpia bromoides & 9 & 8 & 4 & 4 & 2 \\
\hline
\end{tabular}

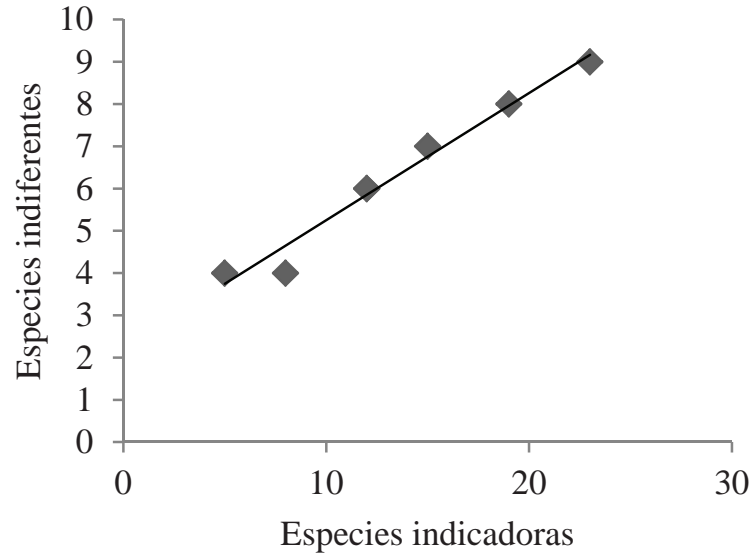

Figura 3. Análisis de regresión entre el número de especies indiferentes y el total de especies indicadoras $(r=0,98, P=0,0001)$. Regression analysis between the number of indifference species and the total indicators species $(r=0.98, P=0.0001)$.

dios de nitrógeno en el suelo (cuadro 6). Los valores promedios para los factores temperatura, reacción y humedad descendieron al considerar la cobertura en mayor proporción que aquellos que subieron, alcanzando las diferencias a 0,54, 0,19 y 0,21 respectivamente, lo que caracteriza los biotopos más fríos, ácidos (con deficiencia de nutrientes) y algo secos.

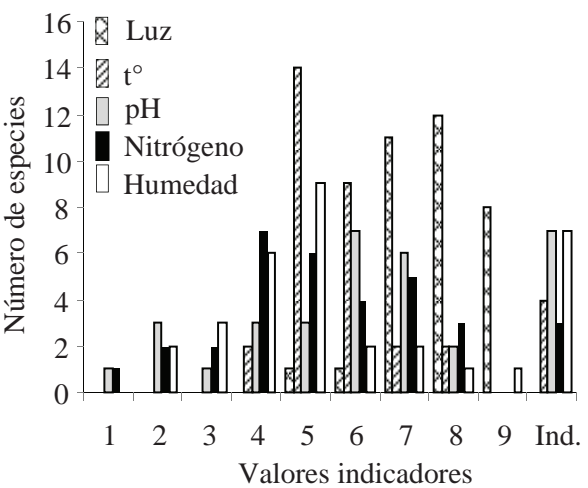

Figura 4. Número total de especies indicadoras en cada tramo de la escala para los factores de luz, temperatura $\left(\mathrm{t}^{\circ}\right)$, reacción $(\mathrm{R})$, nitrógeno $(\mathrm{N})$ y humedad $\left(\mathrm{H}_{2} \mathrm{O}\right)$ en todas las comunidades.

Total number of indicator species in each section of the scale for factors of light, temperature $\left(\mathrm{t}^{\circ}\right)$, reaction $(\mathrm{R})$, nitrogen $(\mathrm{N})$ and moisture $\left(\mathrm{H}_{2} \mathrm{O}\right)$ in all communities.

Al considerar la cobertura el promedio del valor indicador de luz fue de 7,90 con una variación muy baja que alcanzó a 4,96 \%, aunque mayor que la calculada solo con la presencia de especies para el mismo factor. El valor máximo $(8,67)$ para luminosidad se encontró en la pradera de cadillo-aira y el menor $(7,38)$ en la pradera de chépicacadillo, la primera es una pradera totalmente expuesta y la 
Cuadro 5. Promedios de los diferentes valores indicadores para las comunidades según el número de especies. Average of different indicator values for communities depending on the number of species.

\begin{tabular}{lccccc}
\hline Comunidad / Factor: & Luz & Temperatura & Reacción & Nitrógeno & Agua \\
\hline Matorral de ñirre & 7,67 & 5,50 & 4,63 & 4,45 & 4,64 \\
Silvopastoreo con ñirre & 7,40 & 4,75 & 4,00 & 5,20 & 5,25 \\
Silvopastoreo con chacay & 7,80 & 5,42 & 5,36 & 5,14 & 4,71 \\
Pradera de trébol-ballica & 7,74 & 6,63 & 4,71 & 4,67 & 4,89 \\
Pradera de chépica-cadillo & 7,83 & 5,40 & 5,17 & 5,18 & 4,84 \\
Pradera de cadillo-aira & 8,13 & 5,50 & 4,00 & 4,25 & 4,25 \\
Estepa de cadillo & 8,17 & 5,44 & 4,13 & 4,50 & 4,20 \\
Promedio & 7,82 & 5,52 & 4,57 & 4,77 & 4,68 \\
Desviación estándar & 0,27 & 0,56 & 0,56 & 0,40 & 0,37 \\
Coeficiente de variación (\%) & 3,40 & 10,08 & 12,15 & 8,32 & 7,86 \\
\hline
\end{tabular}

Cuadro 6. Promedios de los diferentes valores indicadores para las comunidades según la cobertura de las especies. Average of different indicator values for communities depending on the coverage of species.

\begin{tabular}{lccccc}
\hline Comunidad / Factor: & Luz & Temperatura & Reacción & Nitrógeno & Agua \\
\hline Matorral de ñirre & 7,74 & 5,48 & 5,19 & 4,22 & 5,20 \\
Silvopastoreo con ñirre & 7,95 & 5,00 & 5,90 & 6,51 & 5,05 \\
Silvopastoreo con chacay & 7,74 & 3,55 & 6,27 & 6,01 & 5,14 \\
Pradera de trébol-ballica & 7,88 & 5,30 & 5,85 & 6,00 & 3,94 \\
Pradera de chépica-cadillo & 7,38 & 4,91 & 2,02 & 4,76 & 4,89 \\
Pradera de cadillo-aira & 8,67 & 5,87 & 2,53 & 2,34 & 2,73 \\
Estepa de cadillo & 7,96 & 4,78 & 2,92 & 4,57 & 4,36 \\
Promedio & 7,90 & 4,98 & 4,38 & 4,92 & 4,47 \\
Desviación estándar & 0,39 & 0,73 & 1,82 & 1,42 & 0,90 \\
Coeficiente de variación (\%) & 4,96 & 14,73 & 41,47 & 28,98 & 20,03
\end{tabular}

segunda crece en lugares con árboles aislados de Nothofagus dombeyi. El valor promedio de los indicadores de temperatura bajó mucho, llegando a 4,98, con un máximo de 5,87 en la pradera de chépica-cadillo y un mínimo de 3,55 en el silvopastoreo con chacay, este último valor se corresponde con los biótopos deprimidos con mayor amplitud térmica diaria que ocupa esta comunidad. La variación alcanzó al 14,73\%.

El valor promedio indicador para la reacción del suelo $(\mathrm{pH})$ bajó al considerar la cobertura a 4,38, con la mayor variación de 41,47 \% lo que indica una gran oscilación de los valores que presentaron un máximo de 6,27 en el silvopastoreo con chacay y un mínimo de 2,02 en la pradera de cadillo-aira. Los valores calculados indican que este sería el más variable en las distintas comunidades y por ello, probablemente el mejor diferenciador de los diferentes manejos. Como se dijo anteriormente el valor indicador de nitrógeno presentó un incremento en relación a los calcu- lados con la sola presencia de las especies. El promedio de este indicador alcanzó a 4,92 con una variación de 28,98 $\%$. El máximo valor $(6,51)$ se presentó en silvopastoreo con ñirre y el mínimo $(2,34)$ en la pradera de cadillo-aira, es decir, en las mismas comunidades donde se habían presentado al considerar las especies. El valor indicador de humedad presentó un promedio de 4,47 descendiendo con respecto al obtenido con la presencia de los indicadores, la variación de este promedio fue alta, alcanzando a 20,03 \%. En este caso el valor máximo $(5,20)$ se obtuvo en el matorral de ñirre y el menor $(2,73)$ en la pradera de cadillo-aira.

Distribución de las especies. Los valores promedios de las especies se concentran en los tramos más altos de la escala de luz, lo que caracteriza a todas las comunidades como heliófilas, pero el vértice más alto lo presenta la pradera de chépica-cadillo, mientras que el más bajo, el silvopastoreo con ñirre (figura 5). Las curvas de temperatura tienden a 

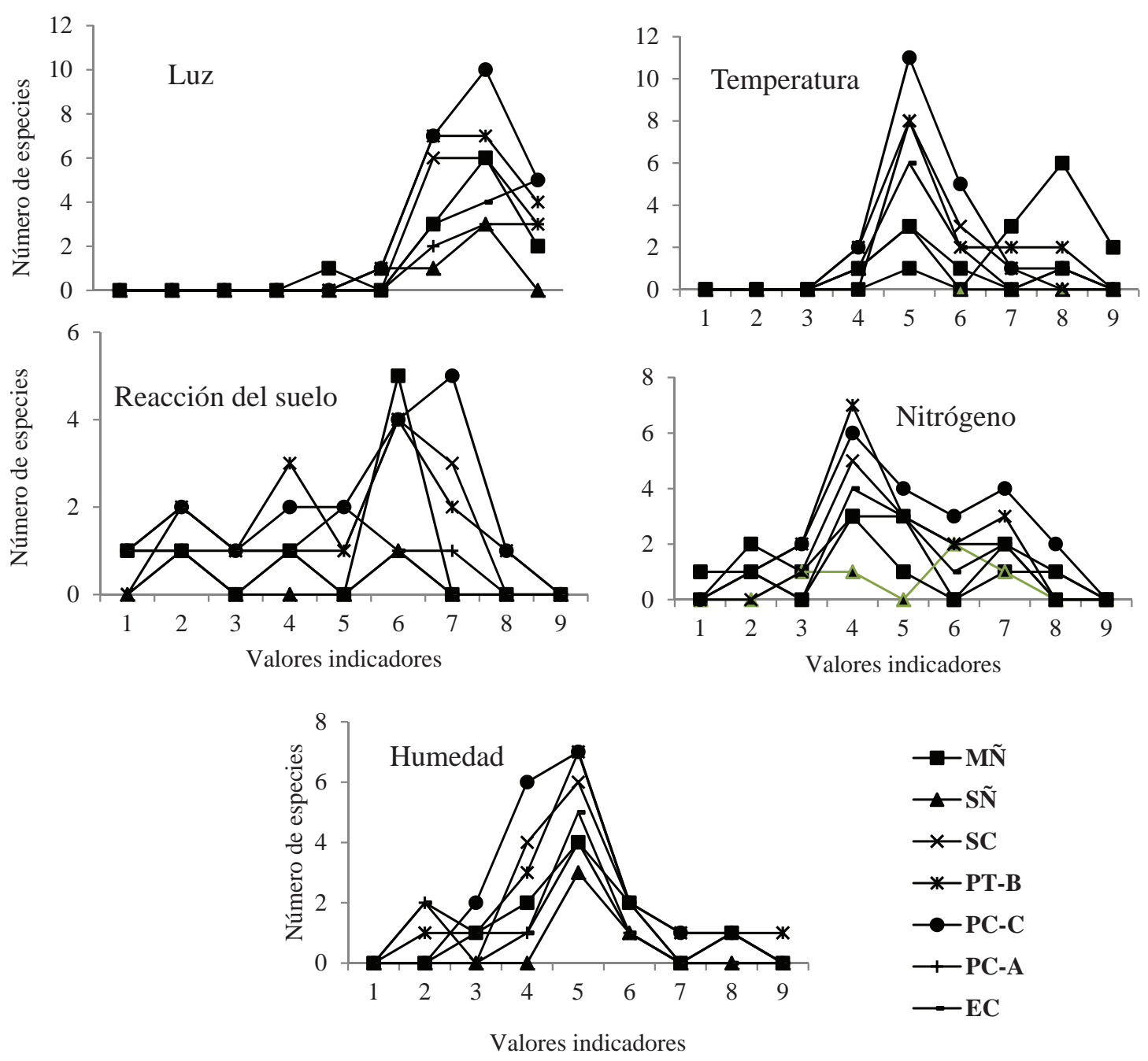

Figura 5. Distribución del número de especies indicadoras para los factores de luz, temperatura, reacción, nitrógeno y humedad por cada tramo de la escala en las distintas comunidades (abreviaturas en el cuadro 2).

Distribution of the number of indicator species for the factors light, temperature, reaction, nitrogen and moisture for each step of the scale in different communities (abbreviations in table 2).

ser bimodales, con un máximo en el valor intermedio de 5 y otro vértice más bajo, en el tramo 8 , aquí nuevamente la pradera de chépica-cadillo alcanza los valores más altos. Las curvas del número de especies distribuidas en los tramos del factor indicador reacción del suelo tienden a presentar una mayor amplitud con especies en todos los tramos, aunque en 6 y 7 se ubican los vértices de la mayoría de las comunidades (figura 5). Los valores más altos se concentran en el matorral de ñirre y en la pradera de chépica-cadillo. También las curvas de especies para el valor indicador de nitrógeno tienden a distribuirse en todos los tramos de la escala con vértices claros en los valores 2, 4 y 7, teniendo el intermedio la mayor cantidad de especies. Lo anterior puede interpretarse como una mayor variación de este factor en los suelos de las distintas comunidades. En este caso, los mayores valores se presentan en las comunidades de praderas de ballica-trébol y de chépica-cadillo.
Las curvas de especies para el factor humedad tienden a concentrarse en los valores 4 y 5 lo que indica características xéricas en las comunidades (figura 5). Nuevamente las praderas de trébol-ballica y de chépica-cadillo presentan los vértices más altos.

Distribución de las coberturas. Al graficar la cobertura (abundancia) de las especies en los diferentes tramos de la escala del valor indicador de luz también los vértices de ellas se concentran en los tramos más altos confirmando el carácter de heliófilo para las comunidades (figura 6). Aquí se presentaron tres vértices importantes: el primero, en el tramo 7 correspondiente a la pradera de chépica-cadillo; el segundo, en el tramo 8, ocupado por el silvopastoreo en ñirre y el tercero, en el 9 formado únicamente por la pradera de cadillo-aira. Lo anterior coincide con lo presentado por las curvas de especies. Las curvas de temperatura 

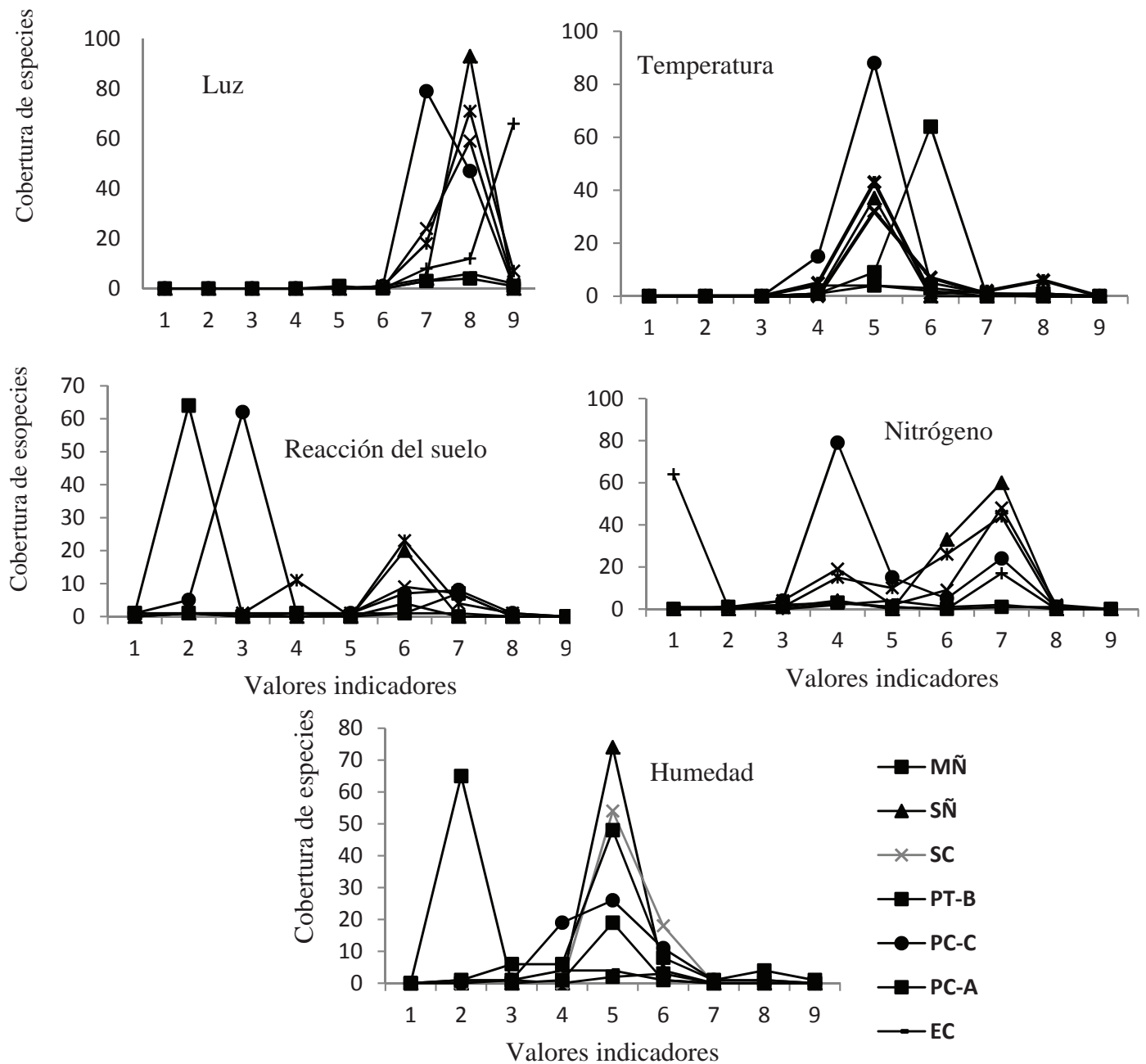

Figura 6. Distribución de la cobertura de especies indicadoras para los factores de luz, temperatura, reacción, nitrógeno y humedad por cada tramo de la escala en las distintas comunidades (abreviaturas en el cuadro 2).

Distribution of coverage indicator species for the factors light, temperature, reaction, nitrogen and moisture for each step of the scale in different communities (Abbreviations in table 2).

presentan una mayor concentración utilizando la cobertura con dos vértices claros, uno en el valor 5 dominado por la pradera de chépica-cadillo y otro en el valor 6, en la pradera de cadillo-aira.

Las curvas de cobertura de las especies distribuidas en los indicadores de reacción del suelo $(\mathrm{pH})$ trasladan sus vértices a los valores más bajos de la escala, 2 y 3 con las praderas de cadillo-aira y de chépica-cadillo respectivamente (figura 6). Hay un tercer vértice más bajo en el tramo 6 donde se reúnen varias comunidades. En este caso, las curvas tienden a concentrarse más que al considerar sólo las especies. En estas curvas queda demostrada la pérdida de nutrientes del suelo principalmente en praderas lo que disminuye el $\mathrm{pH}$. Las curvas de cobertura en los valores indicadores de nitrógeno, tienen una dispersión muy distinta a la encontrada sólo con las especies, ya que destacan dos comunidades, la pradera de cadillo-aira con un vértice en el valor 1, es decir, hábitats muy pobres en nitrógeno y la pradera de chépica-cadillo con su vértice más alto en el valor 4 de suelos algo deficientes en nitrógeno. En este valor indicador se concentraba la mayoría de las comunidades al considerar sólo la presencia de especies indicadoras. El valor 7 es ocupado por varias comunidades aunque con valores de cobertura más bajos, destacan aquí el silvopastoreo con ñirre, el silvopastoreo con chacay y la pradera de trébol-ballica, comunidades con altos niveles de nitrógeno en el suelo, lo cual está de acuerdo con un mayor pastoreo. Por último, las curvas de cobertura para el valor de humedad del suelo, presentan un vértice semejante a las obtenidas con la presencia de especies en el valor 5 que indica suelos frescos, pero la pradera de cadillo-aira forma un vértice en el tramo 2 presentándose como muy seca (figura 6).

Especies indiferentes. Ninguna especie se mostró indiferente al factor luz lo que demuestra la importancia de él para los vegetales (cuadro 7). El factor temperatura pre- 
Cuadro 7. Número de especies indiferentes a cada factor en las comunidades. Los números entre paréntesis indican porcentaje de cobertura de las especies en la comunidad.

Number of indifferent species to each factor in the communities. Numbers in parentheses indicate cover percentage of species in the community.

\begin{tabular}{lcccc}
\hline Asociación / Factor & Temperatura & Reacción & Nitrógeno & Agua \\
\hline Matorral de ñirre & $2(1)$ & $4(4)$ & $1(1)$ & $2(1)$ \\
Silvopastoreo con ñirre & $1(60)$ & $3(77)$ & 0 & $1(60)$ \\
Silvopastoreo con chacay & $3(44)$ & $4(65)$ & $1(1)$ & $2(4)$ \\
Pradera de trébol-ballica & $3(39)$ & $5(55)$ & $1(1)$ & $2(22)$ \\
Pradera de chépica-cadillo & $3(19)$ & $5(44)$ & $1(1)$ & $470)$ \\
Pradera de cadillo-aira & $2(11)$ & $3(12)$ & 0 & 0 \\
Estepa de cadillo & $2(2)$ & $5(6)$ & 0 & $2(1)$ \\
\hline
\end{tabular}

sentó una especie indiferente en el silvopastoreo con ñirre, dos en el matorral de ñirre, en la pradera de chépica y en la estepa de cadillo. El silvopastoreo con chacay y las praderas de trébol-ballica y de chépica-cadillo presentaron tres especies indiferentes para este factor. Considerando la cobertura el silvopastoreo con ñirre resultó la comunidad con mayor peso de especies indiferentes, también son altos los del silvopastoreo con chacay y los de la pradera de trébol ballica, menor peso presentan estas especies en las praderas de chépica-cadillo y de cadillo-aira. En el matorral de ñire (comunidad inicial de la sucesión secundaria) y en la estepa de cadillo (comunidad terminal) las especies indiferentes tienen escasa cobertura y por ello, poca importancia.

El carácter indicador de reacción del suelo presentó la mayor cantidad de especies indiferentes, llegando a tres en el silvopastoreo con ñirre y en la pradera de cadilloaira, a cuatro en el matorral de ñirre y en el silvopastoreo con chacay y a cinco en las praderas de trébol-ballica, de chépica-cadillo y en la estepa de cadillo. Con excepción de las especies indiferentes de las dos comunidades extremas (inicial y terminal de la sucesión secundaria) que presentaron bajos valores de cobertura, todas las otras comunidades presentaron alta cobertura aunque variable. Las mayores coberturas de las especies indiferentes se presentan en los dos silvopastoreos con $67 \%$ y $65 \%$, respectivamente. El factor nitrógeno no presenta especies indiferentes en el silvopastoreo con ñirre, en la pradera de cadillo-aira y en la estepa de cadillo (cuadro 7). El resto de las comunidades presentó una sola especie indiferente a este factor. El factor humedad del suelo, resultó muy variable en el número de especies indiferentes por comunidad, en cuatro comunidades se presentaron dos especies indiferentes, en una (matorral de ñirre) se presentó solo una y ninguna en la pradera de cadillo-aira. Sólo la pradera de chépica-cadillo presentó cuatro especies indiferentes al factor humedad, lo que podría afectar los resultados por la alta cobertura (70 $\%$ ) de ellas. La cobertura de estas especies indiferentes es alta sólo en el silvopastoreo con ñirre y en la pradera de chépica-cadillo, 60 \% y $70 \%$, respectivamente.
Comparación con la sucesión secundaria. En la serie de degradación el indicador de luz aumenta siguiendo el curso de la degradación hasta la pradera de cadillo-chépica para disminuir un poco en la estepa de cadillo, situación terminal (figura 7). El indicador de temperatura se mantiene más o menos estable en valores cercanos a 5 hasta la pradera de trébol-ballica, luego aumenta en la pradera de cadillo-aira, seguramente ante una mayor insolación, pero vuelve a bajar en la estepa de cadillo al parecer por un mayor sombreamiento del suelo. La temperatura se mantiene baja en el silvopastoreo con chacay y en la pradera de chépica-cadillo por el sombreamiento del chacay, en el primer caso y por árboles aislados de coihue, en el segundo. El valor indicador de reacción del suelo $(\mathrm{pH})$ aumenta levemente en el silvopastoreo con ñire y en la pradera de trébol-ballica, para descender bruscamente en las dos comunidades de mayor degradación, la pradera de cadillo-aira y la estepa de cadillo, indicando pérdida de nutrientes. Este mismo valor resultó ser el más alto calculado en el silvopastoreo con chacay, pero vuelve a descender con valores muy bajos, en la pradera de chépica-cadillo, demostrando una mayor degradación química del suelo. El indicador nitrógeno, sube con el pastoreo en el silvopastoreo con ñirre y en la pradera de trébolballica, indicando la influencia del ganado, pero disminuye drásticamente en las dos comunidades de mayor degradación, la pradera de cadillo-aira y la estepa de cadillo. La humedad del suelo se mantiene en valores intermedios en el matorral y en silvopastoreo de ñirre, pero desciende en la pradera de trébol-ballica y en la pradera de cadillo-aira, para recuperarse en la estepa de cadillo. El comportamiento de este indicador se asemeja mucho a la temperatura. El indicador humedad muestra valores medios y semejantes en el silvopastoreo en chacay y en la pradera de chépica-cadillo.

Propiedades de los suelos. El pH (en agua y en CaCl) de los suelos de la serie de degradación fue más alto en el silvopastoreo en ñirre y menor en la pradera fresca de trébol-ballica, que incluso fue inferior al presentado por las comunidades degradas pradera de cadillo-aira y estepa de cadillo (cuadro 8). El comportamiento en las dos comuni- 


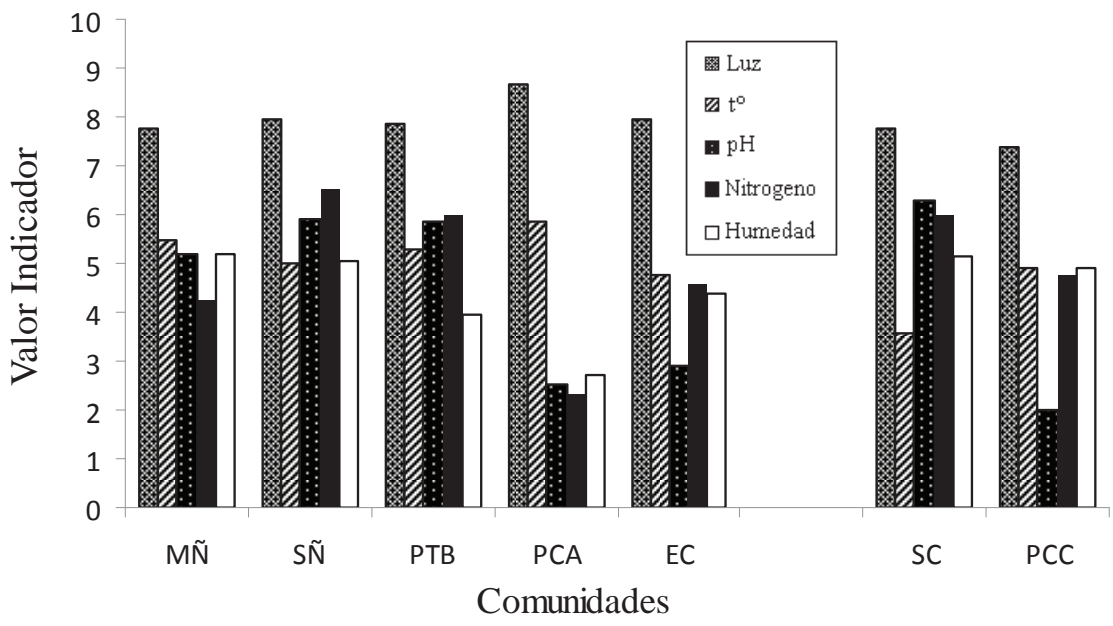

Figura 7. Valor indicador promedio por cobertura para los factores de luz, temperatura $\left(\mathrm{t}^{\circ}\right)$, reacción $(\mathrm{R})$, Nitrógeno $(\mathrm{N})$ y Humedad $\left(\mathrm{H}_{2} \mathrm{O}\right)$ para cada comunidad estudiada.

Average indicator value of the coverage for the factors light, temperature $\left(t^{\circ}\right)$, reaction $(\mathrm{R})$, nitrogen $(\mathrm{N})$ and humidity (H2O) for each community studied.

Cuadro 8. Variables edáficas medidas en muestras de suelo de cada comunidad. Las 5 primeras columnas representan la serie directa de degradación, las últimas 2, a las comunidades que se desvían de la serie. Significado de las abreviaturas en el cuadro 2.

Soil variables measured in soil samples from each community. The first 5 columns represent the direct series of degradation; the last 2 are communities that deviate from the series. Abbreviations in table 2 .

\begin{tabular}{|c|c|c|c|c|c|c|c|}
\hline Variables / Comunidades: & MÑ & $\mathrm{SN}$ & PT-B & PA-C & EC & SC & PC-C \\
\hline $\mathrm{pH}$ en agua $(1: 2,5)$ & 6,2 & 6,7 & 5,3 & 6,4 & 6,3 & 6,5 & 5,3 \\
\hline $\mathrm{pH}$ en $\mathrm{CaCl}_{2} 0,01 \mathrm{M}$ & 5,7 & 6,0 & 4,6 & 5,8 & 5,8 & 6,1 & 4,7 \\
\hline Materia orgánica (\%) & 17,7 & 23,9 & 29,9 & 19,0 & 8,7 & 23,5 & 25,9 \\
\hline $\mathrm{N}$-mineral $\left(\mathrm{N}, \mathrm{NO}_{3}, \mathrm{NH}_{4}\right)\left(\mathrm{mg} \mathrm{kg}^{-1}\right)$ & 40,6 & 28,0 & 215,6 & 75,6 & 39,2 & 72,8 & 56,0 \\
\hline Fósforo Olsen $\left(\mathrm{m} \mathrm{kg}^{-1}\right)$ & 123,8 & 8,6 & 8,7 & 6,5 & 8,4 & 7,9 & 10,2 \\
\hline Calcio intercambiable $\left(\mathrm{cmol} / \mathrm{kg}^{-1}\right)$ & 24,11 & 29,7 & 16,67 & 21,41 & 10,92 & 37,6 & 17,13 \\
\hline
\end{tabular}

dades desviadas de la sucesión directa, silvopastoreo en chacay y pradera de chépica-cadillo fue del mismo tipo, siendo mayor el valor en el primero y reduciéndose en el segundo caso. La materia orgánica aumentó paulatinamente desde el matorral de ñirre a la pradera de trébol-ballica, donde alcanzó su valor máximo (29,9 \%) y luego descendió drásticamente en la pradera de cadillo-aira y en la estepa de cadillo, indicando una fuerte degradación del suelo. También en el caso de las comunidades no consideradas directamente en la serie de degradación presentó un mayor valor la pradera de chépica-cadillo, superando al silvopastoreo en chacay. El nitrógeno inorgánico también alcanzó su valor máximo en la pradera de trébol-ballica, siendo marcadamente menor en los otros manejos. El comportamiento de este nutriente fue errático en las comunidades de silvopastoreo en chacay y pradera de chépica-cadillo. El empobrecimiento en nutrientes minerales en la serie de degradación se ve claramente al considerar los valores de fósforo y de calcio que fueron mayores en el matorral de ñirre, disminuyendo paulatinamente en las otras comunidades de acuerdo a su posición en la serie.

\section{DISCUSIÓN}

Los valores indicadores de las distintas comunidades reflejan muy bien los manejos que las han originado $\mathrm{y}$, además, muestran el grado de alteración en que se encuentran sus hábitats desde el punto de vista microclimático y también edáfico, como lo plantearan Ersten et al. (1998) y Schaffers y Sykora (2000), lo que confirma la utilidad de ellos para caracterizar y diferenciar manejos silvoagropecuarios, que causan distintos tipos y grados de alteración sobre los ecosistemas (Ellies et al. 1993). Lo más importante es que esta evaluación cuantitativa y representativa de la realidad, se lleva a cabo utilizando malezas como bioindicadores sin derroche de esfuerzo y dinero. Estos 
bioindicadores vegetales son abundantes y se encuentran en casi todos los ecosistemas del centro y sur de Chile, incluso en comunidades boscosas nativas (Toledo 2007).

Los bioindicadores vegetales utilizados en este trabajo se concentran en aquellos factores climáticos, como luz y temperatura; mientras en factores edáficos como $\mathrm{pH}$, nitrógeno y humedad, tienden a ocupar toda la escala. Por ello, estos últimos son importantes para diferenciar comunidades próximas en el tiempo y espacio, como la serie de degradación investigada (Ramírez et al. 1995). Los dos primeros factores son climáticos y dependen del macroclima; mientras que los otros indican características del suelo, generalmente alteradas por la intervención humana, como son $\mathrm{pH}$, nutrientes y eutrofización, entre otros.

El factor menos importante para discriminar el estado de degradación del hábitat fue la luz, de hecho ella presentó valores altos y semejantes en todos los manejos, lo que por un lado está de acuerdo con los ecosistemas abiertos y poco estratificados trabajados y por otro, con el hecho de que las especies indicadoras son malezas adaptadas a los altos requerimientos de luz de las plantas de cultivo. El factor temperatura discriminó parcialmente los manejos estudiados, como la luz este factor también depende más del macroclima que de condiciones propias del hábitat.

Los factores indicadores de condiciones edáficas son más importantes para distinguir la degradación de los biótopos, entre ellos destaca la reacción del suelo $(\mathrm{pH})$ que osciló entre valores muy ácidos a intermedios, en este amplio rango se pueden segregar los distintos manejos de acuerdo a la pérdida de nutrientes y de materia orgánica en el suelo, lo que se traduce en una menor fertilidad, valores medianos por el contrario, estarán más próximos a la situación original con un suelo orgánico de mayor fertilidad y a veces eutrofización, por el aporte de fecas del ganado, como se visualizó en la pradera fresca de trébol-ballica (Ramírez et al. 1995). Los menores valores se calcularon para los manejos más degradados donde el suelo erosionado presenta baja cobertura vegetal. Los valores de nitrógeno también presentaron una alta variabilidad entre los diferentes manejos, fueron más altos en los silvopastoreos y pradera de trébol-ballica, si se considera el menor valor alcanzado por el matorral de ñirre, inicio de la degradación, se puede ver el efecto beneficioso sobre la fertilidad del suelo que aporta el ganado en los primeros estadios de intervención, pero posteriormente, estos vuelven a descender por la pérdida de suelo orgánico y de cobertura vegetal por erosión. Por último, el factor humedad, aunque presentó variabilidad media, no tiene mucha importancia en la distinción del grado de degradación de los manejos, pero queda claro que las condiciones hídricas son intermedias en los manejos iniciales, para luego reducirse como consecuencia de la compactación del suelo, que altera el sistema poroso y la pérdida de materia orgánica, que es útil para retener humedad, estas condiciones se observan claramente en los bajos valores de la pradera de cadillo-aira (Steubing y Ramírez 1995).
Normalmente, los trabajos sobre indicadores biológicos vegetales de otras latitudes, tienden a considerar todas las especies como indicadoras (Ramírez et al. 1992), ya que existen los antecedentes y estudios para colocarlas en uno de los tramos de los factores luz, temperatura, reacción del suelo, nitrógeno y humedad, sin embargo, se comprobó que aunque no todas las especies de una comunidad sean indicadoras, también se pueden estudiar sus condiciones de sitio, seleccionando aquellas que tienen antecedentes suficientes para actuar como tal, el resultado se vuelve más real, cuando la cobertura de las mismas es mayor como sucede en este caso con los indicadores de reacción del suelo.

Con los análisis estadísticos multivariados fue posible descubrir algunas especies nativas que podrían actuar como indicadoras, por lo cual sería importante realizar estudios de sus requerimientos, los que complementados con la literatura, podrían servir para aumentar el catálogo de especies indicadoras del centro-sur de Chile preparado por Ramírez et al. (1991). En este sentido, son muy útiles las interpretaciones de los gradientes ecológicos de los dos primeros ejes, más importantes, en los análisis de componentes principales y aunque se basan en el conocimiento de especies reconocidas como tal, permiten ubicar aquellas de posición intermedia poco conocidas o desconocidas en sus requerimientos de sitio. Un caso interesante de este estudio lo constituye el subarbusto, Acaena integerrima, que siendo una planta nativa, comienza a colonizar terrenos erosionados y descubiertos de vegetación, adquiriendo con ello el carácter de pionera, con un valor indicador de degradación del suelo muy marcado. Sería importante poder darle valores como indicador ecológico, ya que ella resiste las condiciones edáficas, que llegan a su máximo en la estepa terminal que le da el nombre.

En este estudio sólo se pudo incluir una especie leñosa como indicadora Rosa rubiginosa, pero en los estadios primarios de una serie de sucesión secundaria de degradación como la estudiada en este trabajo, estas formas de vida son importantes, especialmente, aquellas pioneras del género Nothofagus, por ello sería útil reunir los datos de la literatura existente sobre los requerimientos de sitio de nuestros árboles autóctonos (Weinberger 1997, Weinberger y Ramírez 2001, Vidal et al. 2011) para proceder a darles sus correspondientes valores indicadores en la escala de Ellenberg.

El efecto de sombreamiento de arbustos o árboles aislados sobre los estratos inferiores donde crecen la mayoría de las hierbas indicadores, se refleja claramente en las comunidades algo desviadas de la serie de sucesión directa, silvopastoreo con chacay y pradera de chépica-cadillo. De acuerdo a lo anterior, sería importante estudiar especies nativas capaces de indicar falta de luz pero en los tramos más inferiores del indicador, ya que ellas son muy necesarias para la discriminación de manejos, seguramente en el interior de bosques sombríos y en el estrato herbáceo de matorrales donde aparecen algunas como: Pilea elliptica Hook.f., P. elegans Gay, Chrysosplenium valdivianum 
Hook. d PL suff y Rhamnus diffusus Clos., entre otras (Steubing et al. 1979).

Con el análisis de valores indicadores en la serie de sucesión secundaria descrita por Ramírez et al. (2012) queda de manifiesto que ella tiene su origen en la diferente intensidad del pastoreo en las distintas comunidades y que, además, la interpretación dada por esos autores en cuanto al lugar que ocupan en la serie, correspondería a la realidad, incluso, en aquellas dos comunidades que se desvían de la sucesión original. Aunque en las comunidades secundarias más pastoreadas tiende a aumentar la temperatura como respuesta a una disminución de la cubierta vegetal y una mayor insolación del suelo, esta vuelve a disminuir en la estepa que representaría la comunidad terminal de la sucesión, posiblemente por el aumento de plantas nativas arbustivas que vuelven a recuperar el espacio libre dejado por las malezas introducidas ante la degradación del suelo.

Los análisis de los suelos coinciden con la evaluación lograda con los indicadores ecológicos, ya que ambos muestran un enriquecimiento de la materia orgánica y nitrógeno mineral en la pradera fresca, debido a la influencia del ganado, que aporta a ambos parámetros con sus heces, lo que en la pradera de trébol-ballica incluye eutrofización del suelo.

\section{AGRADECIMIENTOS}

Investigación realizada en el marco del Proyecto CA 012.05 UACh-HYDROAISEN.

\section{REFERENCIAS}

Álvarez M, BM Möseler, C San Martín. 2013. Tendency and distribution indices for ordinal variables applied to Ellenberg indicator values. Gayana Botanica 70(2): 225-233.

Ellenberg H, H Weber, R Döll, V. Wirth, W Werner, D Paulsen. 1992. Zeigerwerte von Pflanzen in Mittel Europas. Scripta Geobotanica 18: 5-258.

Ellies A, C Ramírez, R Mac Donald.1993. Cambios en la porosidad de un suelo por efecto de su uso. Turrialba 43(1): 77-82.

Ersten ACD, JRM Alkemade, MJ Wassen.1998. Calibrating Ellenberg indicator values for moisture, acidity, nutrient avaibility and salinity in the Netherlands. Plant Ecology 135: 113-124.

Hill MO, DB Roy, JO Mountfort, RGH Bunce. 2000. Extending Ellenberg's indicator values to a new area: an algoriyhmic approach. Journal of applied Ecology 37: 3-15.
Leyer I, K Wesche. 2007. Multivariate statistik in der Ökologie. Berlin, Deutschland. Springer. 221 p.

Ramírez C, V Finot, C San Martín, A Ellies. 1991. El valor indicador ecológico de las malezas del Centro-Sur de Chile. Agro Sur 19(2): 94-116.

Ramírez C, C San Martín, V Finot, D Ríos. 1992. Evaluación de praderas usando indicadores ecológicos. Agro Sur 20(2): 85-100.

Ramírez C, C San Martín, V Finot, A Ellies. 1995. Diferenciación de manejos agropecuarios en un suelo trumao (andisol) usando indicadores ecológicos. Ciencia e Investigación Agraria 22(1-2): 3-14.

Ramírez C, V Sandoval, C San Martín, M Álvarez, Y Pérez, C Novoa. 2012. El paisaje rural antropogénico de Aisén: Estructura y dinámica de la vegetación. Gayana Botanica 69(1): 219-231.

San Martín C, J Villagra, C Novoa. 2009. Comparación de manejos pratenses del centro-sur de Chile utilizando valores bioindicadores de Ellenberg. Gayana Botanica 66(2): 158170.

Schafers AP, KV Sykora. 2000. Reliability of Ellenberg indicator values for moisture, nitrogen and soil reaction: a comparison with field measurements. Journal of Vegetation Science 11: 225-244.

Steubing L, C Ramírez, M Alberdi. 1979. Artenzusammensetzung, Lichtgenuss und Energiegehalt der Krautschicht des valdivianischen Regenwaldes bei St. Martín. Vegetatio 39(1) 25-33.

Steubing L, C Ramírez. 1995. Vegetationsanalyse eines Fussballfeldes in Valdivia (Chile). Schriftenreihe für Vegetationskunde 27: 217-221.

Toledo G. 2007. Potenciales plantas invasoras de los bosques nativos en el centro-sur de Chile. Tesis Licenciatura en Ciencias. Valdivia, Chile. Facultad de Ciencias, Universidad Austral de Chile. 71 p.

Vidal O, JR Bannister, V Sandoval, Y Pérez, C Ramírez. 2011. Woodland communities in the Chilean cold-temperate zone (Baker and Pascua basins): Floristic composition and morpho-ecological transition. Gayana Botanica 68(2): 141-154.

Weinberger P. 1997. Definición de grupos ecológicos en formaciones boscosas siempreverdes de la zona austral de Chile. Bosque 18(2): 29-41.

Weinberger P, C Ramírez. 2001. Microclima y regeneración natural de Raulí, Roble y Coigüe (Nothofagus alpina, N. obliqua y N. dombeyi). Bosque 22(1): 11-26.

Wikum D, GF Shanholtzer. 1978. Application of the Braun-Blanquet cover-abundance scale for vegetation analysis in land development. Environmental Management 2(4): 323-329.

Zuloaga F, O Morrone, M Belgrano. 2008. Catálogo de las plantas vasculares del cono sur. Missouri, USA. Botanical Garden Press. 3348 p. 